ARTICLE

https://doi.org/10.1038/s41467-019-12018-7

\title{
Controlled release of hydrogen isotope compounds and tunneling effect in the heterogeneously- catalyzed formic acid dehydrogenation
}

\author{
Kohsuke Mori (i] ${ }^{1,2}$, Yuya Futamura1, Shinya Masuda', Hisayoshi Kobayashi ${ }^{3}$ \& Hiromi Yamashita ${ }^{1,2}$
}

\begin{abstract}
The hydrogen isotope deuterium is widely used in the synthesis of isotopically-labeled compounds and in the fabrication of semiconductors and optical fibers. However, the facile production of deuterium gas $\left(D_{2}\right)$ and hydrogen deuteride $(H D)$ in a controlled manner is a challenging task, and rational heterogeneously-catalyzed protocols are still lacking. Herein, we demonstrate the selective production of hydrogen isotope compounds from a combination of formic acid and $\mathrm{D}_{2} \mathrm{O}$, through cooperative action by a PdAg nanocatalyst on a silica substrate whose surface is modified with amine groups. In this process, $D_{2}$ is predominantly evolved by the assist of weakly basic amine moieties, while nanocatalyst particles in the vicinity of strongly basic amine groups promote the preferential formation of HD. Kinetic data and calculations based on semi-classically corrected transition state theory coupled with density functional theory suggest that quantum tunneling dominates the hydrogen/deuterium exchange reaction over the metallic PdAg surfaces.
\end{abstract}

\footnotetext{
${ }^{1}$ Division of Materials and Manufacturing Science, Graduate School of Engineering, Osaka University, 2-1 Yamada-oka, Suita, Osaka 565-0871, Japan.

${ }^{2}$ Elements Strategy Initiative for Catalysts Batteries ESICB, Kyoto University, Katsura, Kyoto 615-8520, Japan. ${ }^{3}$ Kyoto Institute of Technology, Matsugasaki, Sakyo-ku, Kyoto 606-8585, Japan. Correspondence and requests for materials should be addressed to K.M. (email: mori@mat.eng.osaka-u.ac.jp) or to H.Y. (email: yamashita@mat.eng.osaka-u.ac.jp)
} 
ydrogen isotopes, such as deuterium gas $\left(\mathrm{D}_{2}\right)$ and hydrogen deuteride (HD), are minor components of naturally occurring molecular hydrogen, and have applications as fine chemicals owing to their unique physical, quantum, and nuclear properties ${ }^{1}$. In particular, $\mathrm{D}_{2}$ is often employed for the synthesis of a diverse array of deuterium-labeled compounds, which have been recognized as indispensable research tools in the life, environmental, and material sciences because of their non-radioactive and stable characteristics ${ }^{2-6}$. Unfortunately, commercially available $\mathrm{D}_{2}$ gas is quite expensive, since it is produced by the electrolysis of $\mathrm{D}_{2} \mathrm{O}$ with the investment of large amounts of energy. The reactions of various metals, including $\mathrm{Na}$, $\mathrm{Fe}$, and $\mathrm{Mg}$, with $\mathrm{D}_{2} \mathrm{O}$ have also been traditionally employed to generate this gas on a laboratory scale, but these reagents have serious drawbacks in that they are toxic, produce large amounts of waste, and require high reaction temperatures ${ }^{7}$.

A number of metal-catalyzed $\mathrm{H} / \mathrm{D}$ exchange reactions between $\mathrm{H}_{2}$ and $\mathrm{D}_{2} \mathrm{O}$, especially those based on $\mathrm{Ir}^{8}, \mathrm{Rh}^{9,10}$, and $\mathrm{Ru}^{11,12}$, have been developed based on the investigation of hydrogenase enzymes $^{13}$. Sajiki and co-workers ${ }^{14}$ explored $\mathrm{Pd} / \mathrm{C}$-catalyzed $\mathrm{D}_{2}$ generation via $\mathrm{H} / \mathrm{D}$ exchange reactions using a $\mathrm{H}_{2} / \mathrm{D}_{2} \mathrm{O}$ combination, and applied the $\mathrm{D}_{2}$ that was generated in situ to the synthesis of D-labeled compounds. More recently, Fujita and coworkers ${ }^{15}$ developed a catalytic method to produce $\mathrm{D}_{2}$ from deuterated methanol $\left(\mathrm{CD}_{3} \mathrm{OD}\right)$ and $\mathrm{D}_{2} \mathrm{O}$ using an $\mathrm{Ir}$ complex bearing functional bipyridonate ligands in the presence of sodium hydroxide.

Formic acid ( $\mathrm{HCOOH}, \mathrm{FA})$ has been the subject of significant research effort because it is one of the most promising hydrogen storage materials, due to its low toxicity, nonflammable liquid state at room temperature, and high hydrogen content $\left(43.8 \mathrm{~g} \mathrm{H}_{2}\right.$ $\mathrm{kg}^{-1}$ or $\left.52 \mathrm{~g} \mathrm{H}_{2} \mathrm{~L}^{-1}\right)^{16-18}$. Systems involving FA can also allow economical $\mathrm{CO}_{2}$-mediated hydrogen storage energy cycling via the regeneration of FA through the hydrogenation of $\mathrm{CO}_{2}{ }^{19-22}$. Driven by these intrinsic advantages, numerous investigations have been conducted with the aim of identifying highly active catalysts for the dehydrogenation of FA, which has resulted in relatively sophisticated systems intended for industrial applications ${ }^{23,24} \cdot \mathrm{H}_{2}$ can be controllably released through the thermodynamically favored dehydrogenation of $\mathrm{FA}(\mathrm{HCOOH} \rightarrow$ $\mathrm{H}_{2}+\mathrm{CO}_{2}, \Delta G=-48.4 \mathrm{~kJ} \mathrm{~mol}^{-1}$ ) in the presence of suitable catalysts. However, in such systems, complete suppression of the side reaction $\left(\mathrm{HCOOH} \rightarrow \mathrm{CO}+\mathrm{H}_{2} \mathrm{O}, \Delta G=-28.5 \mathrm{~kJ} \mathrm{~mol}^{-1}\right.$ ) is required, because of the potential for catalyst inhibition by $\mathrm{CO}$ poisoning.

Interestingly, hydrogen isotope compounds have been evolved via the $H / D$ exchange reaction during the metal-catalyzed dehydrogenation of FA in $\mathrm{D}_{2} \mathrm{O}$. In an early study, Puddephatt and co-workers ${ }^{25}$ demonstrated that a mixture of $\mathrm{H}_{2}, \mathrm{HD}$, and $\mathrm{D}_{2}$ gases in a molar ratio of 1:2:1 can be generated by reactions between HCOOD and $\mathrm{D}_{2} \mathrm{O}$ or between DCOOH and $\mathrm{H}_{2} \mathrm{O}$, catalyzed by a binuclear $\mathrm{Ru}$ complex. Subsequently, the distribution of $\mathrm{D}$ during the decomposition of deuterated FA analogs (i.e., HCOOD, DCOOH, and DCOOD) in $\mathrm{D}_{2} \mathrm{O}$ in the presence of $\left[\mathrm{Rh}^{\mathrm{III}}\left(\mathrm{Cp}^{\star}\right)(\mathrm{bpy})\left(\mathrm{H}_{2} \mathrm{O}\right)\right]^{2+}$ and the heterodinuclear iridium-ruthenium complex $\left[\mathrm{Ir}^{\mathrm{III}}\left(\mathrm{Cp}^{*}\right)\left(\mathrm{H}_{2} \mathrm{O}\right)(\mathrm{bpm}) \mathrm{Ru}^{\mathrm{II}}(\mathrm{bpy})_{2}\right]$ $\left(\mathrm{SO}_{4}\right)_{2} \quad\left(\mathrm{Cp}^{*}=\right.$ pentamethylcyclopentadienyl, bpm $=2,2^{\prime}$-bipyrimidine, bpy $=2,2^{\prime}$-bipyridine) was explored by Fukuzumi et al. ${ }^{26,27}$. A convenient means of producing $\mathrm{D}_{2}$ and various deuterated compounds using a half-sandwich Ir complex with $4,4^{\prime}$-dihydroxy-2,2' -bipyridine ligands was also demonstrated by Himeda and co-workers ${ }^{28}$. Although some progress has been achieved in conjunction with the use of transition metal complexes, these processes often require high catalyst concentrations and expensive deuterated FA to obtain reasonable levels of activity and selectivity. In addition, the use of homogeneous catalysts on an industrial scale is extremely challenging due to the difficulties involved in recovering the precious metals and ligands from the reaction mixture. For these reasons, systems based on heterogeneous catalysts in association with undeuterated FA and $\mathrm{D}_{2} \mathrm{O}$ would be highly beneficial, but such processes have not yet been researched, in spite of the obvious practical advantages.

Our group has previously reported that the presence of basic functional groups on support materials in the vicinity of active Pdbased alloy nanoparticles (NPs) promotes the production of highpurity $\mathrm{H}_{2}$ during the dehydrogenation of $\mathrm{FA}^{29-34}$. Herein, we describe a straightforward method for the production of hydrogen isotope compounds from a combination of $\mathrm{FA}$ and $\mathrm{D}_{2} \mathrm{O}$, using a $\mathrm{PdAg}$ nanocatalyst on a substrate that has been surface modified with amine groups. This work also demonstrates that the selectivity of this process can be tuned by varying the basicity of the amine moieties. The $H / D$ exchange reactions between $F A$ and $\mathrm{D}_{2} \mathrm{O}$ is found to involve a quantum tunneling effect, as evidenced by the large kinetic isotope effect (KIE) value, and based on semiclassically corrected transition state theory (SC-TST) coupled with density functional theory (DFT) calculations.

\section{Results}

Structural Characterization. Various amine-grafted mesoporous silica (SBA-15) substrates (termed SBA-15-Amine-x (SA-x), where $x=1-5)$ were synthesized using a surface modification technique. This silica possesses relatively large hexagonal channels $\sim 9 \mathrm{~nm}$ in diameter. The surface modification was performed using a post-synthesis method in association with silane-coupling reagents containing different amine groups, as shown in Fig. 1. The SA-4 specimen, having Schiff base functional groups $(-\mathrm{N}=$ $\mathrm{CH}_{2}$ ), was also synthesized by the further modification of SA-2 with formaldehyde. These substrates were impregnated with $\mathrm{Pd}$ and $\mathrm{Ag}(1 \mathrm{wt} \% \mathrm{Pd}$; Pd:Ag molar ratio = 1:1) simply by immersion in aqueous solutions of $\mathrm{Pd}\left(\mathrm{NH}_{3}\right)_{4} \mathrm{Cl}_{2}$ and $\mathrm{AgNO}_{3}$ followed by reduction with $\mathrm{NaBH}_{4}$. The resulting catalysts are denoted $\mathrm{PdAg} /$ SA- $x \quad(x=1-5)$ herein. The concentration of grafted amine functionalities in all samples was determined to be $\sim 0.3 \mathrm{mmol} \mathrm{g}^{-1}$ substrate using thermogravimetric (TG) analysis. The presence of the amine groups was confirmed by the analysis of each specimen by Fourier transform-infrared (FT-IR) spectroscopy (Supplementary Fig. 1) and N $1 s$ X-ray photoelectron spectroscopy (XPS) (Supplementary Fig. 2). The long-range ordering of hexagonally packed mesoporous structures in these materials was also demonstrated on the basis of low-angle X-ray diffraction (XRD) patterns (Supplementary Fig. 3).

The textural properties of the PdAg/SA- $x$ as well as $\mathrm{PdAg} / \mathrm{S}$ made with unmodified silica are summarized in Table S1. All samples generated $\mathrm{N}_{2}$ adsorption/desorption isotherms having characteristic type IV curves with sharp capillary condensation steps and H1-type hysteresis loops. These features indicate highly ordered and well-preserved cylindrical mesopores (Supplementary Fig. 4). The Brunauer-Emmett-Teller $(\mathrm{BET})$ surface areas $\left(S_{\mathrm{BET}}\right)$, pore diameters $\left(D_{\text {pore }}\right)$ and pore volumes $\left(V_{\mathrm{p}}\right)$ for the members of the PdAg/SA- $x$ series were all found to be slightly lower than those for the unmodified PdAg/S. During surface modification, the silane-coupling reagents were able to readily diffuse through the channels, which ensured homogeneous grafting, because the estimated molecular sizes of these reagents (up to $1 \mathrm{~nm}$ ) were significantly smaller than the diameters of the channels.

Supplementary Figure 5 presents high-angle annular dark-field scanning transmission electron microscopy (HAADF-STEM) images together with size distribution plots for the $\mathrm{PdAg} / \mathrm{S}$ and PdAg/SA- $x$ materials. The PdAg NPs on the PdAg/SA- $x$ were evidently well dispersed and had a narrow size distribution, with average diameters in the range of $4.5-7.3 \mathrm{~nm}$. These values are 

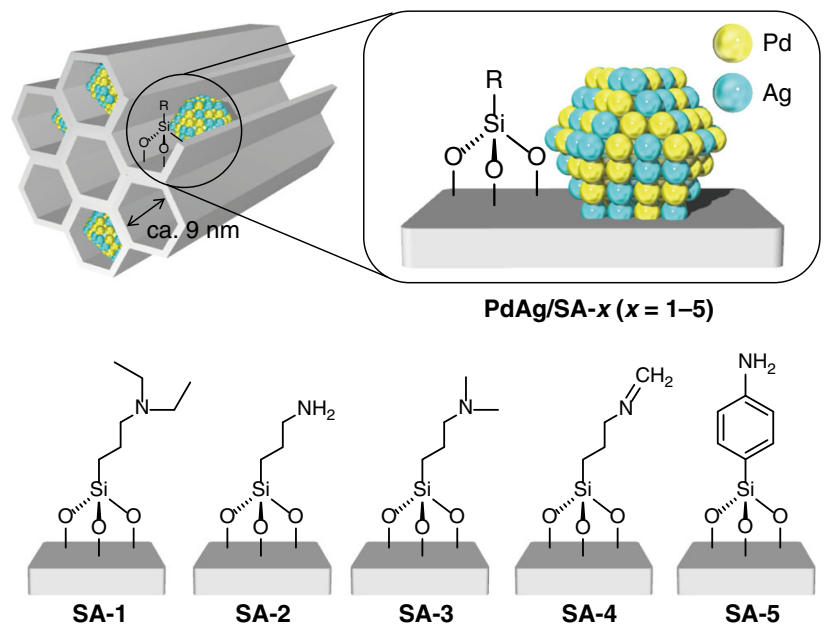

Fig. 1 Schematic illustrations of catalysts. The PdAg nanoparticles were deposited on the surface of SBA-15 mesoporous silica modified with silanecoupling reagents containing different amine groups (termed SA-x, where $x=1-5$ )

smaller than the $7.5 \mathrm{~nm}$ NP size on the unmodified PdAg/S, and so amine functionalization evidently resulted in the formation of smaller NPs. This result indicates that variations in the basicity of the grafted amine groups may affect both the formation of $\mathrm{Pd}$ metal nuclei and the consecutive growth, ultimately producing different NP sizes.

The shapes of the Pd K-edge X-ray absorption near edge structure (XANES) spectra generated by the PdAg/SA- $x$ and unmodified $\mathrm{PdAg} / \mathrm{S}$ differ from that seen in the $\mathrm{PdO}$ spectrum, but resemble the spectrum of Pd foil (Supplementary Fig. 6A). The FT-extended X-ray absorption fine structure (FT-EXAFS) spectra of $\mathrm{PdAg} / \mathrm{SA}-x$ and $\mathrm{PdAg} / \mathrm{S}$ exhibit a single intense peak associated with $\mathrm{Pd}-\mathrm{Pd}$ bonds that suggests the presence of metallic Pd (Supplementary Fig. 6B). However, the Pd-Pd bond lengths for these materials were slightly longer than that for $\mathrm{Pd}$ foil, indicating $\mathrm{Pd}-\mathrm{Ag}$ bonds. The $\mathrm{Ag} \mathrm{K}$-edge XANES spectra obtained from the PdAg/SA- $x$ and $\mathrm{PdAg} / \mathrm{S}$ are similar to that of $\mathrm{Ag}$ foil, and the FT-EXAFS spectra of these specimens exhibit a single sharp peak ascribed to contiguous $\mathrm{Ag}-\mathrm{Ag}$ bonds with lengths of $\sim 2.6-2.8 \AA$ (Supplementary Fig. 7). This peak is shifted to a slightly shorter interatomic distance compared with that for pure Ag foil. These results present clear evidence for the formation of PdAg alloy NPs. Additionally, the Pd $3 d$ peaks in the XPS spectra produced by the PdAg/SA- $x$ are slightly shifted to higher binding energies compared to those for the unmodified sample (Supplementary Fig. 8A). A similar shift of the Ag $3 d$ peaks in the XPS spectra is also evident after modification (Supplementary Fig. 8B). Our experimental results suggest that surface amine functional groups were present at the peripheries of the loaded PdAg NPs to alter their electronic state. The alternation of the electronic state of the supported NPs by the organic modifier of the support materials have also been reported previously ${ }^{33}$.

Controllable release of hydrogen isotope compounds. Utilizing the prepared catalysts having different amine functional groups, the dehydrogenation reaction was conducted in $\mathrm{D}_{2} \mathrm{O}$ solutions containing $5 \mathrm{M}$ HCOOH:HCOONa (9:1) at $343 \mathrm{~K}$ for $30 \mathrm{~min}$. The selectivities for the resulting hydrogen isotope compounds ( $\mathrm{HD}$ and $\mathrm{D}_{2}$ ) are summarized in Fig. 2a, together with the turnover frequency (TOF) values based on the amount of Pd. No
$\mathrm{H}_{2}$ formation was observed, and the molar ratios of total hydrogen isotope compounds to $\mathrm{CO}_{2}$ generated during the course of the reaction were close to 1 for all samples. The use of bare SA5 without PdAg NPs does not show any activity. It should be noted that the present catalytic system limits the undesirable formation of $\mathrm{CO}$ to less than the detection limit of the gas chromatography (GC) system $(<2 \mathrm{ppm})$. As expected, we succeeded in the controlled synthesis of these hydrogen isotope compounds by changing the surface grafted amine groups. As an example, the PdAg/SA-1 (having the strongly basic $-\mathrm{NEt}_{2}$ groups) preferentially generated HD with $65 \%$ selectivity, while $\mathrm{D}_{2}$ formation was predominant with $87 \%$ selectivity when using the PdAg/SA-5, which was modified with the weakly basic $-\mathrm{PhNH}_{2}$ groups. The $\mathrm{D}_{2}$ and $\mathrm{HD}$ selectivity appeared to be independent of the yield level and remained unchanged during the reaction, suggesting that the $\mathrm{H}-\mathrm{D}$ exchange reaction with the produced hydrogen isotope compounds did not take place (Supplementary Fig. 9). In addition, the catalytic activity was also greatly affected by the properties of the supports. As an example, the TOF value obtained with PdAg/SA-5 was $65 \mathrm{~h}^{-1}$, which was seven times that observed using the PdAg/SA-1.

The $\mathrm{H}_{2} \mathrm{O} / \mathrm{D}_{2} \mathrm{O}$ molar ratio in the reaction solution also significantly affected selectivity. Fig. 2b, c plots the selectivity for various hydrogen isotope compounds versus the $\mathrm{D}_{2} \mathrm{O}$ mol\%. In the case of the $\mathrm{PdAg} / \mathrm{SA}-1$, HD selectivity increased with increasing $\mathrm{D}_{2} \mathrm{O}$ concentration, accompanied by a decrease in $\mathrm{H}_{2}$, up to a $\mathrm{D}_{2} \mathrm{O}$ concentration of $67 \mathrm{~mol} \%$, with no $\mathrm{D}_{2}$ formation observed. The maximum HD selectivity of $80 \%$ was attained at $80 \mathrm{~mol} \% \mathrm{D}_{2} \mathrm{O}$ without the formation of $\mathrm{H}_{2}$, after which the HD selectivity decreased. In the reaction with $\mathrm{PdAg} / \mathrm{SA}-5$, the $\mathrm{HD}$ selectivity increased along with the $\mathrm{D}_{2} \mathrm{O}$ mol\%, while the $\mathrm{D}_{2}$ selectivity was drastically enhanced at $\mathrm{D}_{2} \mathrm{O}$ levels above $50 \mathrm{~mol} \%$. The highest $\mathrm{D}_{2}$ selectivity was obtained at $100 \mathrm{~mol} \% \mathrm{D}_{2} \mathrm{O}$, with $87 \%$ selectivity. In contrast, the type of amine functional group, PdAg NP loading, reaction temperature, and ratio of FA to sodium formate did not show significant effect on selectivity (Supplementary Figs. 10-13), demonstrating that these parameters are minor factors in determining the selectivity.

The recyclability is a crucial point to consider when heterogeneous catalysts are employed for industrial applications. After the reaction, the $\operatorname{PdAg} / \mathrm{SA}-x(x=1$ and 5$)$ catalysts were easily separated from the reaction mixture and could be reused with retention of its activity and selectivity; preferential formation of hydrogen isotope compounds could be attained for at least five recycling experiments (Supplementary Fig. 14). More interestingly, the present catalyst provides a simple and efficient heterogeneous catalytic system for the in situ deuteration of organic compounds (Table 1 and Supplementary Fig. 15). As an example, the reaction of diphenylacetylene proceeded smoothly using PdAg/SA-5 and $5 \mathrm{M} \mathrm{HCOOH:HCOONa} \mathrm{(9:1)} \mathrm{solution} \mathrm{in}$ $\mathrm{D}_{2} \mathrm{O}$, giving the deuterated product with $96 \%$ deuterated content in $99 \%$ chemical yield. The expected deuterated products were also obtained with excellent deuterated contents via addition reactions of deuterium to carbon-carbon double bonds and deuterium substitution reactions.

The observed variations in selectivity for hydrogen isotope compounds were assessed by determining the adsorption energy $\left(E_{\mathrm{ad}}\right)$ of FA based on DFT calculations for each SA- $x$. This was done because such values reflect the basicity of the amine functional groups, since the adsorption involves formation of an acid-base pair via $\mathrm{N}-\mathrm{H}$... O hydrogen bonding ${ }^{32}$. As an example, the $E_{\text {ad }}$ over SA-1 with $-\mathrm{N}\left(\mathrm{C}_{2} \mathrm{H}_{5}\right)_{2}$ groups was found to be $-215 \mathrm{~kJ} \mathrm{~mol}^{-1}$, which was substantially stronger than the value of $-120 \mathrm{~kJ} \mathrm{~mol}^{-1}$ over SA-5, having $-\mathrm{PhNH}_{2}$ groups. It is worth noting that a good correlation between the HD selectivity and $E_{\text {ad }}$ was observed. Specifically, the HD selectivity increased with increases in $E_{\text {ad }}$, as shown in Fig. 3a, 

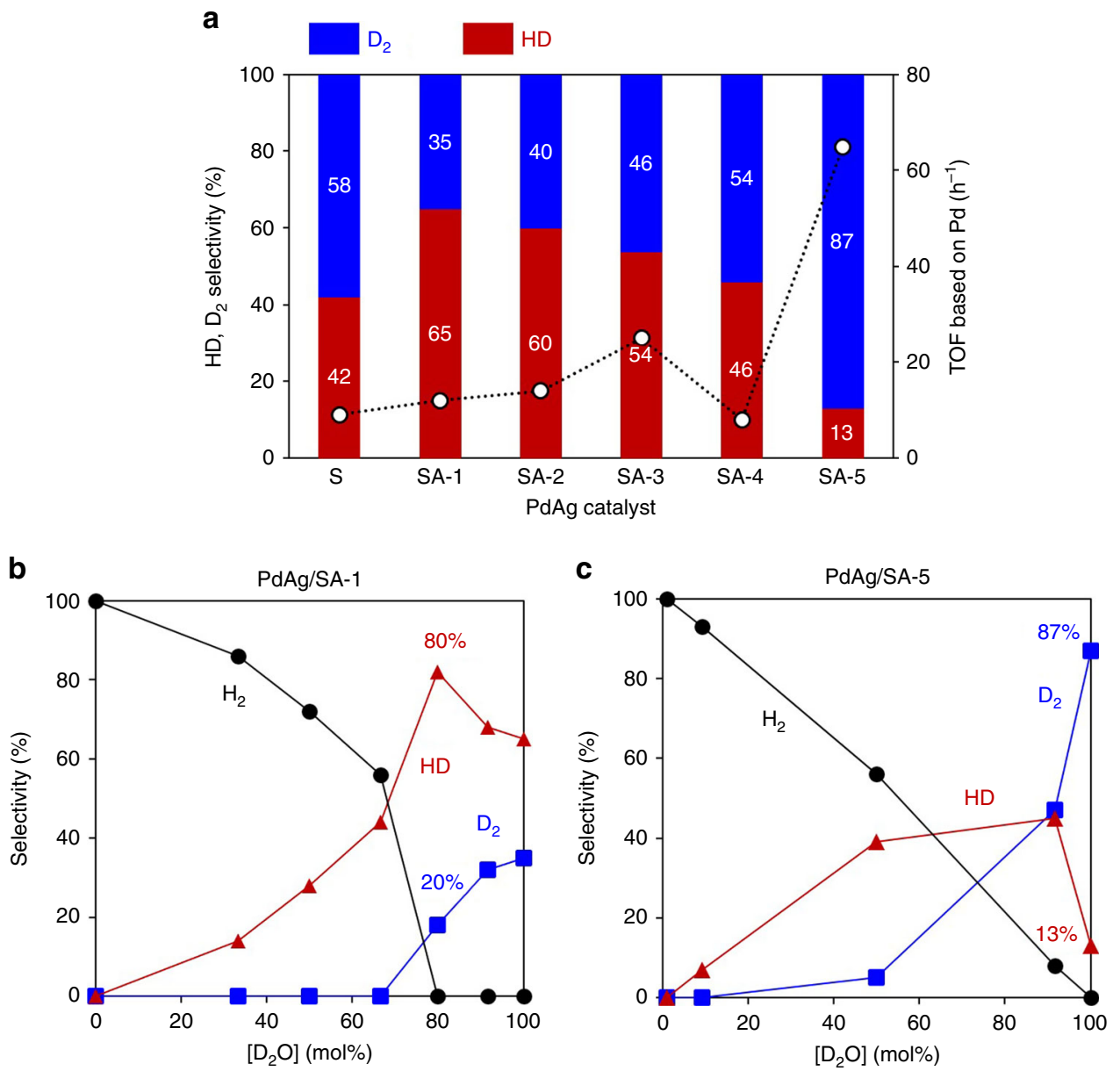

Fig. 2 Selectivity and TOF for the dehydrogenation of FA. a Effect of catalysts using PdAg/S and PdAg/SA-x $(x=1-5)$; b effect of $D_{2} \mathrm{O}$ mol\% in solution using $\mathrm{PdAg} / \mathrm{SA}-1$. c Effect of $\mathrm{D}_{2} \mathrm{O}$ mol\% in solution using $\mathrm{PdAg} / \mathrm{SA}-5$

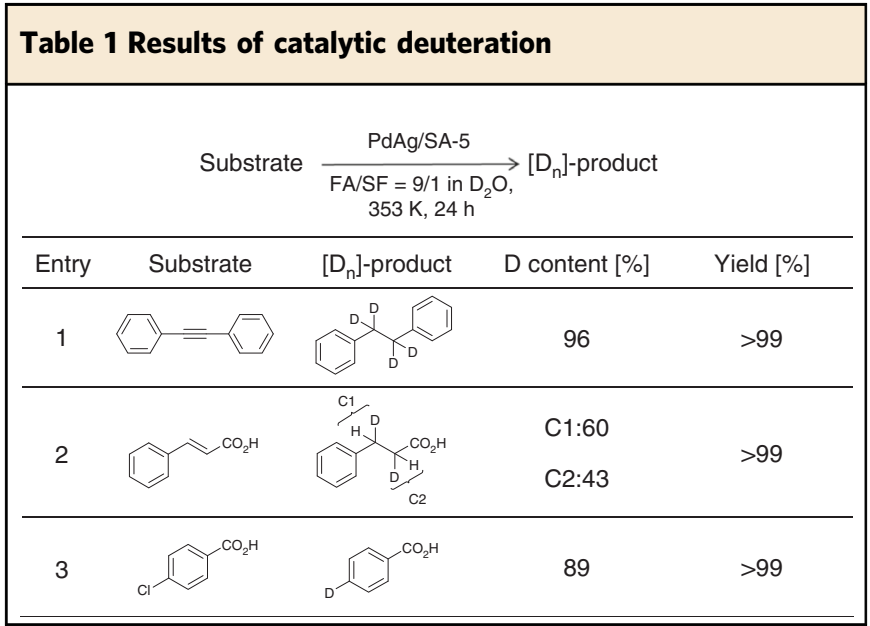

suggesting that the basicity of the amine functional groups plays a crucial role in determining selectivity.

We suspected that differences in the sizes of the PdAg NPs also affected selectivity. However, there was no significant correlation between selectivity and the average diameter of PdAg NPs on the SA- $x$ series of samples, based on HAADF-STEM analysis (Fig. $3 \mathrm{~b})$. Unfortunately, this investigation was unable to accurately decouple the concomitant effect of the basicity of the various amine functional groups from that of the NP size. In order to further examine the effect of NP size on selectivity, a series of colloidal poly(vinylpyrrolidone)-capped Pd NPs were first synthesized under several different conditions and then deposited on the SA $-5^{35}$. The TEM images and size distribution plots shown in Supplementary Fig. 16 demonstrate the fabrication of sizecontrolled $\mathrm{Pd} / \mathrm{SA}-5$ specimens with particle sizes ranging from 2.8 to $16 \mathrm{~nm}$. These materials were subsequently applied to the dehydrogenation of FA in $\mathrm{D}_{2} \mathrm{O}$. As expected, the $\mathrm{D}_{2}$ selectivity was found to remain almost constant at $\sim 90 \%$ regardless of the particle size, although a volcano-type variation in activity based on the particle size was observed, with a maximum TOF at $3.9 \mathrm{~nm}$ (Supplementary Fig. 17).

In addition, Pd-based alloy NPs supported on SA-5 were synthesized, using $\mathrm{Au}, \mathrm{Co}, \mathrm{Cr}, \mathrm{Ni}, \mathrm{Zn}$, or $\mathrm{Cu}$ precursors as the second metal and employing the same impregnation method followed by reduction with $\mathrm{NaBH}_{4}$. Notably, these variations in the metal component did not greatly affect the $\mathrm{D}_{2}$ selectivity (Fig. $3 c)$, although the activity varied significantly. This effect can presumably be ascribed to the synergic effect between the Pd and the second metal, as has been reported previously $29,30,36-38$. These results suggest that the selectivity for the production of hydrogen isotope compounds is governed by the grafted amine groups in the periphery of each active center, and not by the inherent ability of the metal NPs themselves.

Upon consideration of the above results as well as the theoretical studies, we determined a possible reaction pathway 

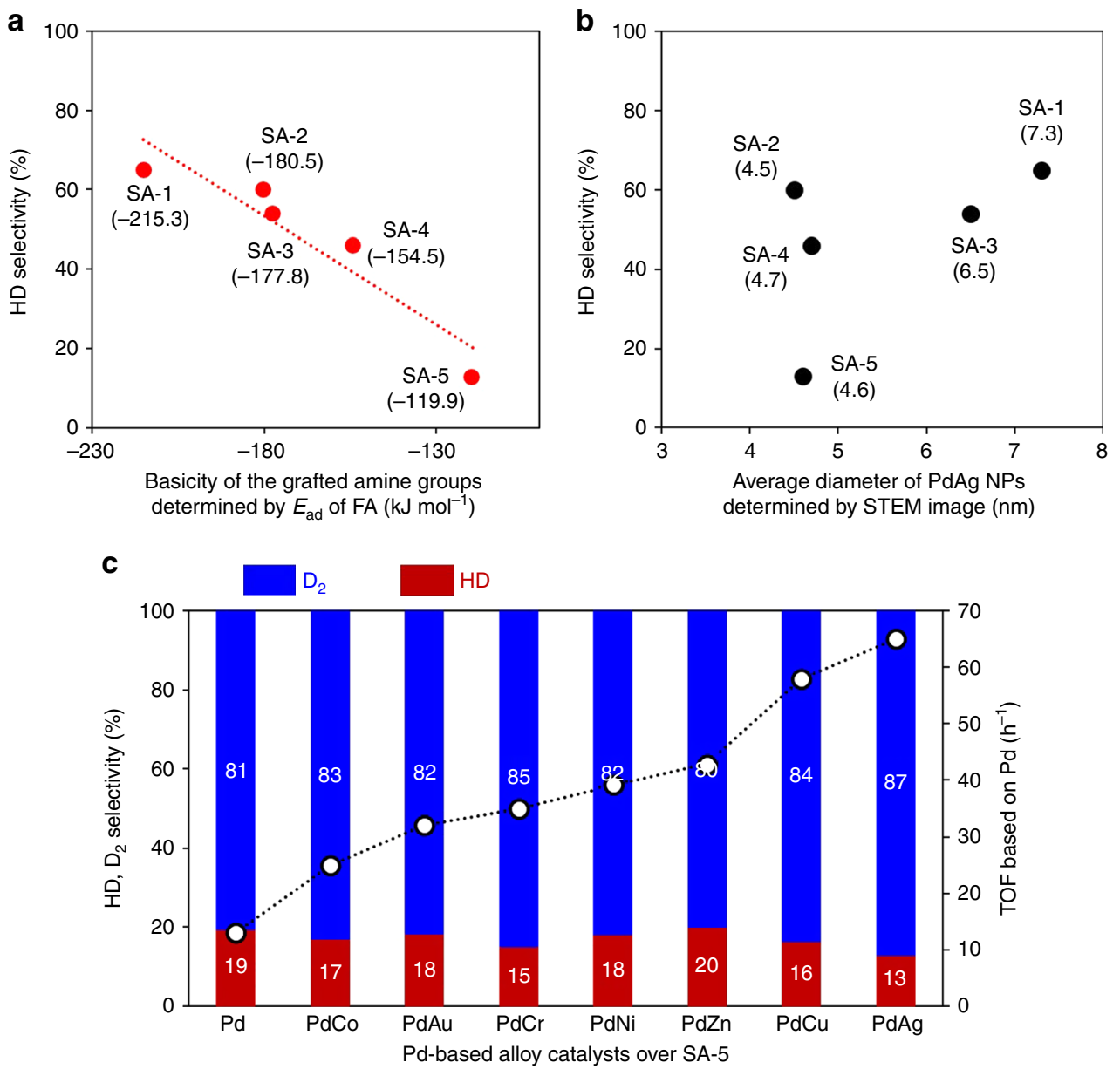

Fig. 3 Key factor for determining selectivity. a Correlations between HD selectivity and basicity of the amine groups determined by the adsorption energy $\left(E_{\mathrm{ad}}\right)$ of $\mathrm{FA} ; \mathbf{b}$ correlations between HD selectivity and the average diameter of PdAg NPs as determined from STEM; c selectivity and TOF values using Pdbased alloy NPs supported on SA-5

leading to the selective formation of hydrogen isotope compounds from the dehydrogenation of $\mathrm{FA}$ in $\mathrm{D}_{2} \mathrm{O}$, as illustrated in Fig. 4. In this process, regardless of the basicity of the amine groups, the reaction of $\mathrm{HCOOH}$ with 1 is promoted by the amine groups in the periphery of the PdAg NPs as a result of the acid-base interaction, affording a Pd formate species along with a proton-scavenged amine group (2). The Pd formate species, for which the trans- $\mathrm{AgO}-\mathrm{PdH}(\mathrm{O})$-bridged configuration is the lowest energy structure, subsequently undergoes isomerization followed by $\beta$-hydride elimination to release $\mathrm{CO}_{2}$ and a Pd hydride species (3). Intermediate 3 undergoes $\mathrm{H}-\mathrm{D}$ exchange reactions in the presence of $\mathrm{D}_{2} \mathrm{O}$ at both the $\mathrm{Pd}$ and basic amine sites, but to differing extents according to the basicity of the amine groups. In the case of PdAg/SA-1, which has strongly basic $-\mathrm{NEt}_{2}$ groups, four preferentially undergoes an $\mathrm{H}-\mathrm{D}$ exchange reaction at the $\mathrm{Pd}$ site with a barrier of $E_{\mathrm{a}}=140.2 \mathrm{~kJ} \mathrm{~mol}^{-1}$ to afford 5. Subsequently, an $\mathrm{H}-\mathrm{D}$ exchange reaction proceeds at the basic amine site with a barrier of $212.5 \mathrm{~kJ} \mathrm{~mol}^{-1}$ to produce 6 . The intermediates 5 and 6 release $\mathrm{HD}$ and $\mathrm{D}_{2}$, respectively. Thus, the substantially higher activation energy for the formation of 6 compared to that for 5 contradict the occurrence of the reversed reaction, which also explains the preferential formation of HD. In contrast, the activation energies for the formations of 8 and 9 from 7 via the $\mathrm{H}-\mathrm{D}$ exchange reaction at $\mathrm{Pd}$ and weakly basic $-\mathrm{PhNH}_{2}$ groups in the case of $\mathrm{PdAg} / \mathrm{SA}-5$ were calculated to be 140.2 and $149.8 \mathrm{~kJ} \mathrm{~mol}^{-1}$, respectively. From an energetic point of view, the $\mathrm{H}-\mathrm{D}$ exchange reaction would therefore be expected to occur simultaneously at both sites, leading to the preferential formation of intermediate 9 and ultimately favoring $\mathrm{D}_{2}$ generation. The $E_{\mathrm{a}}$ for the $\mathrm{H}-\mathrm{D}$ exchange reaction at the strongly basic $-\mathrm{N}\left(\mathrm{C}_{2} \mathrm{H}_{5}\right)_{2}$ site is substantially larger than that at the weakly basic $-\mathrm{PhNH}_{2}$ site, which are reflected by the basicity determined by the $E_{\mathrm{ad}}$ of FA in Fig. 3a; the $E_{\mathrm{a}}$ increased with increasing the $E_{\text {ad }}$ of FA. It can therefore be concluded that the selectivity for hydrogen isotope compounds is determined by the basicity of the surface amine groups, which ultimately influence on the $\mathrm{H}-\mathrm{D}$ exchange reaction at the basic sites in the peripheries of the active centers. In addition, the preliminary DFT calculations determined the $E_{\mathrm{a}}$ for the release of hydrogen from 5 or 6 and 8 or 9 were 52.7 and $13.0 \mathrm{~kJ} \mathrm{~mol}^{-1}$, respectively, indicating rate-determining step is the $\mathrm{H}-\mathrm{D}$ exchange reaction at the basic amine site with $\mathrm{D}_{2} \mathrm{O}$. These results also suggest that hydrogen release is accelerated in the presence of weakly basic amines, which accounts for the higher TOF obtained from PdAg/SA-5 as compared to that for $\mathrm{PdAg} / \mathrm{SA}-1$, as shown in Fig. 2a.

To improve our understanding of this mechanism, H/D KIE values were examined in trials using $\mathrm{H}_{2} \mathrm{O}$ and $\mathrm{D}_{2} \mathrm{O}$. Intriguingly, large KIE values of 10 at $323 \mathrm{~K}$ and 23 at $313 \mathrm{~K}$ were obtained when using PdAg/SA-2 and PdAg/SA-5, respectively. Abnormally large KIE values at room temperature or higher generally indicate the participation of a quantum tunneling effect in a proton transfer reaction, and this can be unambiguously confirmed from the temperature dependence of KIE. Specifically, values for the Arrhenius parameters $E_{\mathrm{a}}(\mathrm{D})-E_{\mathrm{a}}(\mathrm{H}), A_{\mathrm{H}} / A_{\mathrm{D}}$, and $\mathrm{KIE}$ of $>5 \mathrm{~kJ}$ 


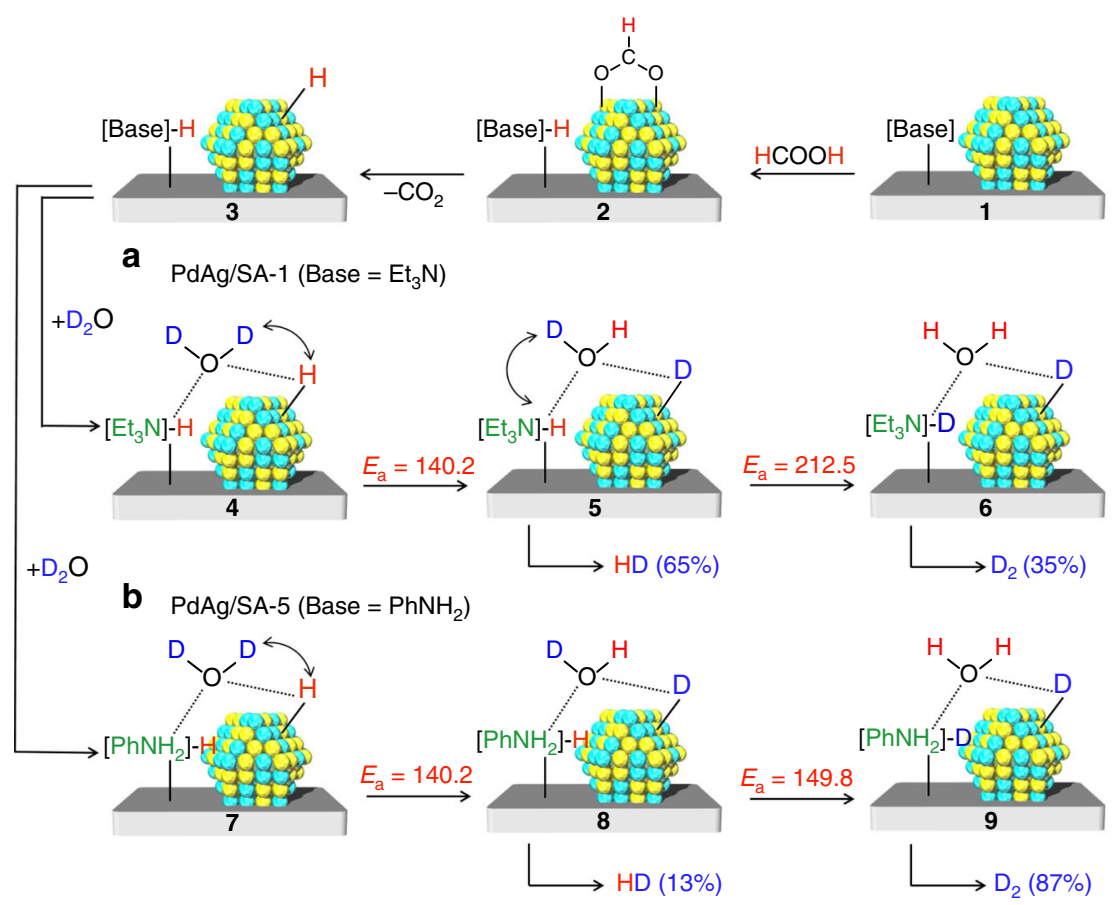

Fig. $4 \mathrm{~A}$ proposed reaction mechanism in $\mathrm{D}_{2} \mathrm{O}$. Pathway (a) represents the selective production of $\mathrm{HD}$ using $\mathrm{PdAg} / \mathrm{SA}-1$ and pathway (b) represents the selective production of $D_{2}$ using $\mathrm{PdAg} / \mathrm{SA}-5$. The values above the arrows are activation energy $\left(E_{\mathrm{a}}\right)\left(\mathrm{kJ} \mathrm{mol}^{-1}\right)$ for each elementary step

$\mathrm{mol}^{-1}, \ll 1$, and $>9$ at $298 \mathrm{~K}$ provide evidence for the involvement of a quantum tunneling effect ${ }^{27,39}$. The Arrhenius plots for $\mathrm{H}_{2} \mathrm{O}$ and $\mathrm{D}_{2} \mathrm{O}$ obtained in the present study are provided in Fig. 5. The resulting $E_{\mathrm{a}}(\mathrm{D})-E_{\mathrm{a}}(\mathrm{H})$ and $A_{\mathrm{H}} / A_{\mathrm{D}}$ values were $19.7 \mathrm{~kJ} \mathrm{~mol}^{-1}$ and 0.005 for PdAg/SA-2 and $9.4 \mathrm{~kJ} \mathrm{~mol}^{-1}$ and 0.06 for PdAg/SA-5, respectively. Our experimental data therefore meet the above requirements, and indicate the contribution of a quantum tunneling effect to the present catalytic cycle.

Tunneling effect calculations. Quantum mechanical tunneling of atoms, which originates from wave-particle duality, has been observed not only in chemical reactions, but also in many other fields of chemistry including astrochemistry and biochemistry ${ }^{40-42}$. In particular, the tunneling of hydrogen species such as protons, hydrogen atoms and hydride ions is an important aspect of acid-base, transfer and proton-coupled electron transfer reactions ${ }^{43-48}$. As an example, hydrogen atoms have been frequently observed to tunnel through various metal surfaces, such as $\mathrm{Cu}(001)^{49}, \mathrm{Ru}(0001)^{50}$ and $\mathrm{Ni}(100)^{51}$, and the motion of hydrogen atoms is known to be enhanced by tunneling at low temperatures. Proton hopping between oxygen atoms on the Brønsted acidic sites of zeolites and heteropolyacids has also been found to be associated with tunneling pathways ${ }^{52,53}$. In addition, it has been demonstrated that reactions based on heterogeneous catalysis, such as $\mathrm{CO}$ oxidation and $\mathrm{NH}_{3}$ formation on metal surfaces, are also affected by tunneling ${ }^{54-58}$.

Atom tunneling can be experimentally demonstrated by constructing a non-linear Arrhenius plot based on data acquired at low temperatures, where the rate constant is independent of temperature, or (as described above) by unusual KIE values. However, the effect of tunneling on reaction rates can also be directly confirmed by theoretical calculations ${ }^{59-62}$. In an effort to theoretically demonstrate the participation of tunneling pathways in the present heterogeneously-catalyzed dehydrogenation of FA using PdAg NPs in the vicinity of amine groups, we adopted the semi-classically corrected TST (SC-TST) developed by Fermann and Auerbach ${ }^{63}$, together with a modification by Bhatia and
Sholl ${ }^{64,65}$. A tunneling crossover temperature, $T_{\mathrm{c}}$, was calculated using the equations

$$
\begin{gathered}
T_{\mathrm{c}}=\frac{h \nu_{\mathrm{Im}}^{\dagger} \Delta E_{\mathrm{ZP}} / k_{\mathrm{B}}}{2 \pi \Delta E_{\mathrm{ZP}}-h \nu_{\mathrm{Im}}^{\dagger} \ln 2}, \\
\Delta E_{\mathrm{ZP}}=\left(E^{\dagger}+\sum_{j=1}^{N-1} \frac{h \nu_{j}^{\dagger}}{2}\right)-\left(E_{\mathrm{A}}+\sum_{i=1}^{N} \frac{h \nu_{i}}{2}\right),
\end{gathered}
$$

where $\Delta E_{\mathrm{ZP}}$ is the activation energy barrier (i.e., the difference in energies between the TS and the local minimum, including the SCF energy $\left(E^{\dagger}\right.$ or $E_{\mathrm{A}}$ ), and the zero-point energy, as defined in Eq. (2)). $N$ is the number of degrees of freedom for the system, $h$ and $k_{\mathrm{B}}$ are the Planck and Boltzmann constants, $v_{1}$ is the harmonic frequency for the local minimum, and $v^{\dagger}$ Im and $v_{j}$ are those for the TS (the former being an imaginary frequency).

Within the SC-TST framework, the reaction rate constant at temperature $T$ is given by Eq. (3), using the TST rate constant, $k^{\text {TST }}(T)$, and the tunneling correction factor, $\Gamma(T)$, which are expressed by Eqs. (4) and (5), respectively. In Eq. (4), $\Delta E$ is the SCF energy difference between the TS and the local minimum.

$$
\begin{gathered}
k^{\mathrm{SC}-\mathrm{TST}}(T)=k^{\mathrm{TST}}(T) \cdot \Gamma(T) \\
k^{\mathrm{TST}}(T)=\frac{\prod_{i=1}^{N} \nu_{j} f\left(h \nu_{i} / 2 k_{\mathrm{B}} T\right)}{\prod_{j=1}^{N-1} \nu_{j}^{\dagger} f\left(h \nu_{j}^{\dagger} / 2 k_{\mathrm{B}} T\right)} \cdot \exp \left(-\frac{\Delta E}{k_{\mathrm{B}} T}\right), f(x)=\frac{\sinh (x)}{x}, \\
\Gamma(T)=\frac{\exp \left(\Delta E_{\mathrm{ZP}} / k_{\mathrm{B}} T\right)}{1+\exp \left(2 \pi \Delta E_{\mathrm{ZP}} / h \nu_{\mathrm{Im}}^{\dagger}\right)}+\frac{1}{2} \int_{-\infty}^{\pi \Delta E_{\mathrm{ZP}} / h \nu_{\mathrm{Im}}^{\dagger}} \mathrm{d} \theta \exp \left(\frac{h \nu_{\mathrm{Im}}^{\dagger} \theta}{\pi k_{\mathrm{B}} T}\right) \operatorname{sech}^{2}(\theta)
\end{gathered}
$$

The production of hydrogen isotope compounds from an FA/ $\mathrm{D}_{2} \mathrm{O}$ combination using PdAg NPs together with amine groups evidently comprises a series of $\mathrm{H}-\mathrm{D}$ exchange reactions between $\mathrm{D}_{2} \mathrm{O}$ and the PdAg NPs and/or amine groups. Here, five 

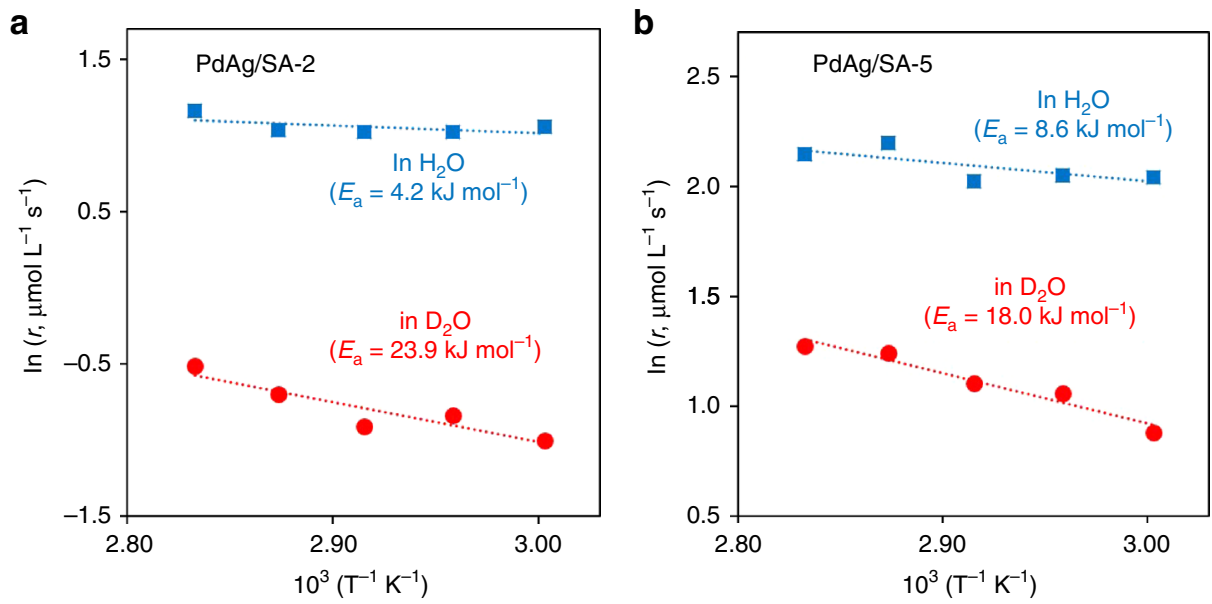

$$
\begin{aligned}
& E_{\mathrm{a}}(\mathrm{D})-E_{\mathrm{a}}(\mathrm{H})=19.7 \mathrm{~kJ} \mathrm{~mol}^{-1} \\
& A_{\mathrm{H}} / A_{\mathrm{D}}=0.005 \\
& \mathrm{KIE}=10 \text { at } 323 \mathrm{~K}
\end{aligned}
$$

$$
\begin{aligned}
& E_{\mathrm{a}}(\mathrm{D})-E_{\mathrm{a}}(\mathrm{H})=9.4 \mathrm{~kJ} \mathrm{~mol}^{-1} \\
& A_{\mathrm{H}} / A_{\mathrm{D}}=0.06 \\
& \mathrm{KIE}=23 \text { at } 313 \mathrm{~K}
\end{aligned}
$$

Fig. 5 Arrhenius plots in $\mathrm{H}_{2} \mathrm{O}$ and $\mathrm{D}_{2} \mathrm{O}$. The obtained Arrhenius parameters were represented for a $\mathrm{PdAg} / \mathrm{SA}-2$ and $\mathbf{b} \mathrm{PdAg} / \mathrm{SA}-5$

representative reaction paths were considered for the calculations according to the above-proposed reaction mechanism. These include (I) direct $\mathrm{H} / \mathrm{D}$ exchange over a PdAg surface, (II) direct $\mathrm{H} / \mathrm{D}$ exchange over an $\mathrm{NEt}_{3}$ group, (III) direct $\mathrm{H} / \mathrm{D}$ exchange over a $\mathrm{PhNH}_{2}$ group, (IV) $\mathrm{H} / \mathrm{D}$ exchange over $\mathrm{PdAg}$ in the vicinity of an $\mathrm{NEt}_{3}$ group, and (V) H/D exchange over PdAg in the vicinity of a $\mathrm{PhNH}_{2}$ group (Fig. 6a).

Table 2 summarizes the $E_{\mathrm{ZP}}, v^{\dagger} \mathrm{Im}$, and $T_{\mathrm{c}}$ values determined via Eq. (1) using a $\mathrm{Pd}_{2} \mathrm{Ag}_{2}$ cluster model. Below these values, quantum tunneling dominates the reaction rates, while above these values classical transitions dominate. The $T_{\mathrm{c}}$ value for path I was determined to be $320 \mathrm{~K}$, which is substantially higher than the values for the other reaction pathways, which range from 123 to $206 \mathrm{~K}$. These values are comparable to those reported for proton-hopping reactions on zeolites $(184-320 \mathrm{~K})^{52}$, but slightly lower than that determined for heteropolyacids $(353 \mathrm{~K})^{53}$. The $k^{\text {TST }}(T)$ and $\Gamma(T)$ values at various temperatures are also provided in Table 2, while Fig. $6 \mathrm{~b}$ presents the Arrhenius plots of $k^{\mathrm{TST}}(T), \Gamma(T)$, and $k^{\mathrm{SC}-\mathrm{TST}}(T)$ values calculated for path I. Above $T_{\mathrm{c}}, k^{\mathrm{SC}-\mathrm{TST}}(T)$, and $k^{\mathrm{TST}}(T)$ are consistent with one another and are also dependent on temperature, meaning that classical transitions dominate. In contrast, $k^{\mathrm{SC}-\mathrm{TST}}(T)$ is almost temperature independent below $T_{c}$ because of the increase in the tunneling correction factor with decreasing temperature. Owing to the quantum tunneling effect, an Arrhenius fit of the rate data affords different activation energies depending on the temperature range investigated. An activation energy of $46.2 \mathrm{~kJ}$ $\mathrm{mol}^{-1}$ was obtained from the $k^{\mathrm{SC}-\mathrm{TST}}(T)$ data between 300 to $400 \mathrm{~K}$, whereas the values of $k^{\mathrm{SC}-\mathrm{TST}}(T)$ from 100 to $200 \mathrm{~K}$ gave an activation energy of $0.8 \mathrm{~kJ} \mathrm{~mol}^{-1}$. It should be noted that $k^{\mathrm{SC}-}$ TST is also larger than $k^{\text {TST }}$ at $T_{\mathrm{c}}$ by a factor of more than $35(\Gamma$ $(320)=35.1)$. Therefore, $T_{c}$ is not a distinct point at which a change in mechanism between quantum tunneling and classical transitions occurs, but rather a point where the relative dominance of the two mechanisms rapidly changes ${ }^{53,63}$. For reaction pathways II-V, the temperature independence of $k^{\mathrm{SC}-\mathrm{TST}}$ (T) suggests that the tunneling mechanism is predominant (Supplementary Fig. 18), although (as noted) the $T_{\mathrm{c}}$ values are lower than those for path I, and are below room temperature. Similar trends are observed in the case of the $\mathrm{Pd}_{7} \mathrm{Ag}_{6}$ cluster model, as summarized in Supplementary Table 3 and Supplementary Fig. 19. Here, the $T_{\mathrm{c}}$ for path I was determined to be 257 $\mathrm{K}$, which is higher than those for paths IV and $\mathrm{V}$. These theoretical investigations strongly suggest that a tunneling mechanism occurs primarily in path I (i.e., direct $H / D$ exchange over the PdAg surface) and plays a crucial role in the overall reaction at temperatures as high as $320 \mathrm{~K}$. The tunneling probability depends to different degrees on the mass of the moving particle, the barrier height, and the barrier width ${ }^{42}$. Especially, the barrier width mainly determines the probability of tunneling in chemical reactions: the narrower a barrier is, the more likely tunneling is ${ }^{41}$. As shown in Supplementary Fig. 20, our calculation results reveal that the $T_{\mathrm{c}}$ correlates well with $v_{\mathrm{Im}}$, not $\Delta E_{\mathrm{ZP}}$. The absolute value $v_{\mathrm{Im}}$ is the curvature at the vicinity of the transition state in the reaction coordinate direction, which significantly influence on the barrier width: the larger a value $v_{\text {Im }}$ is, the narrower a barrier is. The representative reaction paths used to assess the tunneling effect proceed at the same time; thus, the $T_{c}$ of the reaction with highest $T_{c}$ can be experimentally observed. The lower $T_{c}$ in paths II-V suggests that the H-D exchange at the basic amine cites are largely dominated by classical transitions; thus, the effect of basicity of the amine in the $\mathrm{HD} / \mathrm{D}_{2}$ ratio can be experimentally appeared.

\section{Discussion}

We succeeded in the controlled synthesis of hydrogen isotope compounds during the dehydrogenation of $\mathrm{FA}$ in $\mathrm{D}_{2} \mathrm{O}$ by tuning the $\mathrm{H}-\mathrm{D}$ exchange reaction, using specific amine-functionalized PdAg nanocatalysts. The grafted amine groups were found to be indispensable to this process, and the reaction selectivity showed a close correlation with the basicity of the amine groups, with the $\mathrm{D}_{2}$ selectivity increasing with decreasing basicity. This study provides not only advanced insights into the preferred catalyst architecture but also demonstrates an ideal heterogeneous catalyst system with potential applications to this type of transformation. Large KIE values indicative of the quantum tunneling effect was observed for the reaction of FA in $\mathrm{H}_{2} \mathrm{O}$ vs. $\mathrm{D}_{2} \mathrm{O}$. Theoretical investigations also emphasized the involvement of a quantum tunneling mechanism, predominantly during the direct $\mathrm{H}-\mathrm{D}$ exchange reaction over the PdAg surface. 
a

Path I Path II Path III

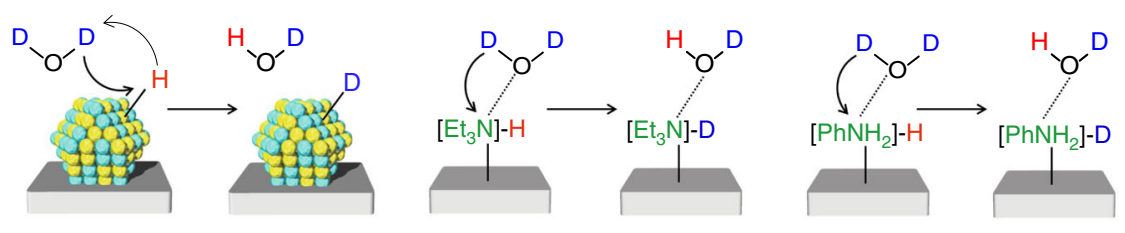

Path IV

Path V
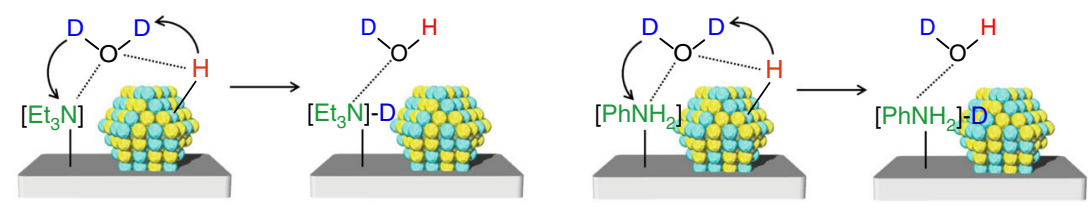

b

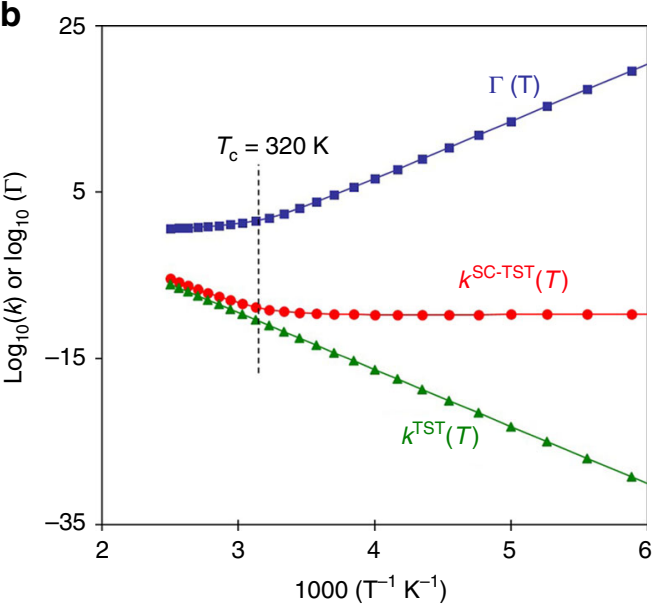

Fig. 6 Tunneling effect calculations. a Reaction paths used to assess the tunneling effect. (I) direct H/D exchange over the PdAg surface, (II) direct H/D exchange on an $\mathrm{NEt}_{3}$ group, (III) direct $\mathrm{H} / \mathrm{D}$ exchange over a $\mathrm{PhNH}_{2}$ group, (IV) $\mathrm{H} / \mathrm{D}$ exchange over $\mathrm{PdAg}$ in the vicinity of an $\mathrm{NEt}_{3}$ group and $\mathrm{V}$ ) $\mathrm{H} / \mathrm{D}$ exchange over $\mathrm{PdAg}$ in the vicinity of a $\mathrm{PhNH}_{2}$ group. b Arrhenius plots as calculated for path I

\begin{tabular}{|llllll|}
\hline \multicolumn{4}{l}{ Table 2 Parameters for the calculation of tunneling effect } \\
& Path I & Path II & Path III & Path IV & Path V \\
\hline$\Delta E_{\mathrm{ZP}}\left(\mathrm{kJ} \mathrm{mol}^{-1}\right)$ & 132.9 & 215.6 & 143.3 & 72.7 & 86.3 \\
$\nu_{\mathrm{lm}}\left(\mathrm{cm}^{-1}\right)$ & 1376.4 & 731.7 & 535.3 & 490 & 886.7 \\
$T_{\mathrm{c}}(\mathrm{K})$ & 320 & 168 & 123 & 113 & 206 \\
\hline
\end{tabular}

\section{Methods}

Materials. $\mathrm{Pd}\left(\mathrm{NH}_{3}\right)_{4} \mathrm{Cl}_{2}$ was obtained from the Sigma-Aldrich Chemical Co. $\mathrm{AgNO}_{3}, \mathrm{FA}$, and 3-aminopropyltriethoxysilane were purchased from Nacalai Tesque, Inc., and aminophenyltrimethoxysilane was obtained from Manchester Organic. All commercially available chemicals were used as received.

Synthesis of the SA-x series. The mesoporous silica support SBA-15 was prepared using a surfactant self-assembly approach as follows: $4.0 \mathrm{~g}$ of Pluronic P123 was dissolved in $30 \mathrm{~g}$ of deionized water and $120 \mathrm{~mL}$ of $2 \mathrm{M} \mathrm{HCl}$ solution with stirring at $313 \mathrm{~K} ; 8.5 \mathrm{~g}$ of TEOS was added into the above solution with stirring for $24 \mathrm{~h}$. The mixture was aged at $353 \mathrm{~K}$ overnight without stirring, and then the product was recovered by vacuum filtration and washed with deionized water. The resulting white powder was dried at $383 \mathrm{~K}$ overnight and calcined at $823 \mathrm{~K}$ for $10 \mathrm{~h}$. Physisorbed water was removed before surface modification by drying the SBA-15 at $403 \mathrm{~K}$ for $3 \mathrm{~h}$ under vacuum. Following this, $100 \mathrm{~mL}$ of a toluene solution containing $1.0 \mathrm{~g}$ of SBA-15 and the appropriate amount of amine-functionalized silane-coupling reagents was stirred at $373 \mathrm{~K}$ for $18 \mathrm{~h}$. The product was recovered by vacuum filtration, washed with toluene and diethyl ether, and then dried under vacuum overnight. An SA-4 sample with Schiff base functional groups was synthesized by the further modification of SA-2 with formaldehyde.
Synthesis of PdAg/SA-x. A quantity of SA-x $(0.3 \mathrm{~g})$ was mixed with $50 \mathrm{~mL}$ of an aqueous solution containing $\mathrm{Pd}\left(\mathrm{NH}_{3}\right)_{4} \mathrm{Cl}_{2}(10 \mathrm{mM}, 2.85 \mathrm{~mL})$ and $\mathrm{AgNO}_{3}(10 \mathrm{mM}$, $2.85 \mathrm{~mL}$ ), followed by stirring at room temperature for $1 \mathrm{~h}$. The suspension was evaporated under vacuum and the resulting powder was dried overnight. Subsequently, each sample was reduced with $\mathrm{NaBH}_{4}$, giving PdAg/SA- $x$ (1 wt\% Pd; Pd: $\mathrm{Ag}$ molar ratio $=1: 1$ ). Analysis using inductively coupled plasma (see Characterization section for details) clearly indicated that the desired amount of each metal species was loaded onto each support.

Characterization. Powder XRD patterns were recorded using a Rigaku Ultima IV diffractometer with $\mathrm{Cu} \mathrm{Ka}$ radiation $(\lambda=1.54056 \AA)$. Nitrogen adsorption-desorption isotherms were acquired at $77 \mathrm{~K}$ using a BELSORP-max system (MicrotracBEL Corp.). Prior to analysis, samples were degassed at $423 \mathrm{~K}$ for $3 \mathrm{~h}$ under vacuum to remove physisorbed water. Specific surface areas were calculated using the BET method based on the nitrogen adsorption data. Inductively coupled plasma optical emission spectrometry data were obtained using a Nippon Jarrell-Ash ICAP-575 Mark II instrument. TG analysis was performed by a Rigaku Thermo plus EVO2 TG8121 system from room temperature to $973 \mathrm{~K}$ at a heating rate of $10 \mathrm{~K} \mathrm{~min}^{-1}$ in air flow $(10 \mathrm{~mL} \mathrm{~min}-1)$. TEM micrographs were obtained with a Hitachi Hf-2000 FE-TEM equipped with a Kevex energy-dispersive X-ray (EDX) detector operated at $200 \mathrm{kV}$. STEM images and elemental maps were obtained with a JEOL-ARM 200F equipped with a Kevex EDX detector (JED$2300 \mathrm{~T}$ ) operated at $200 \mathrm{kV}$. FT-IR spectra were obtained with a JASCO FT/IR6100. Samples were prepared for analysis by mixing with $\mathrm{KBr}$, followed by compression into thin disk-shaped pellets. XPS was performed with a Shimadzu ESCA3400 system, using $\mathrm{Mg} \mathrm{Ka} \mathrm{X}$-ray radiation $(h v=1253.6 \mathrm{eV})$ as the excitation source. The binding energy values for each spectrum were calibrated relative to the contaminant $\mathrm{C} 1 \mathrm{~s}$ core level binding energy of $284.5 \mathrm{eV}$. Pd and Ag K-edge XAFS spectra were acquired using a fluorescence-yield collection technique at the 01B1 beamline at SPring-8, JASRI, Harima, Japan, in conjunction with a Si (111) monochromator (proposal numbers 2018A1144 and 2018B1082). Data reduction was performed using the REX2000 program (Rigaku). 
Dehydrogenation of FA. A $0.1 \mathrm{~g}$ quantity of catalyst was transferred into a $30 \mathrm{~mL}$ reaction vessel containing $1.8 \mathrm{~mL} \mathrm{D}_{2} \mathrm{O}$, after which the vessel was sealed with a rubber septum. The reaction mixture was bubbled with $\mathrm{Ar}$ for $15 \mathrm{~min}$, followed by the addition of $0.2 \mathrm{~mL}$ of a $5 \mathrm{M}$ solution of $\mathrm{HCOOH}: \mathrm{HCOONa}(9: 1)$ in $\mathrm{D}_{2} \mathrm{O}$, with magnetic stirring at $343 \mathrm{~K}$. The amounts of $\mathrm{H}_{2}, \mathrm{HD}$, and $\mathrm{D}_{2}$ generated were determined by GC using a Shimadzu GC-2014 instrument with He as the carrier gas and a Shinwa OGO-SP column held at $77 \mathrm{~K}$, in conjunction with a thermal conductivity detector ${ }^{66}$. TOFs in units of $\mathrm{h}^{-1}$ were calculated as the total moles of all hydrogen isotopes divided by total moles Pd in the catalyst after $30 \mathrm{~min}$.

In situ deuteration in the FA- $\mathbf{D}_{\mathbf{2}} \mathbf{O}$ system. A $0.1 \mathrm{~g}$ quantity of the PdAg/SA-5 was placed into a $30 \mathrm{~mL}$ reaction vessel containing $1.8 \mathrm{~mL} \mathrm{D}_{2} \mathrm{O}$ and $0.1 \mathrm{mmol}$ of the substrate, after which the vessel was sealed with a rubber septum. A $0.2 \mathrm{~mL}$ quantity of a $5 \mathrm{M} \mathrm{HCOOH}: \mathrm{HCOONa}(9: 1)$ solution in $\mathrm{D}_{2} \mathrm{O}$ was added and the reaction mixture was magnetically stirred at $353 \mathrm{~K}$. After $24 \mathrm{~h}$, the reaction mixture was passed through a membrane filter to remove the catalyst. The filtrate was mixed with $\mathrm{Et}_{2} \mathrm{O}(10 \mathrm{~mL})$ and the aqueous layer was extracted with $\mathrm{Et}_{2} \mathrm{O}(2 \times 10$ $\mathrm{mL}$ ), dried over $\mathrm{MgSO}_{4}$, filtered, and concentrated in vacuo to give the analytically pure deuterated produces. The yield was determined by GC/mass spectrometry (MS) equipped with capillary column and the D contents of the products were determined by ${ }^{1} \mathrm{H}$ nuclear magnetic resonance (NMR) spectroscopy on the basis of the integration of the aromatic protons.

Computational method. FA adsorption energy values, $E_{\text {ad }}$, were calculated using DFT, employing the DMol3 program in the Materials Studio 17.2 software pack$\mathrm{age}^{67}$. The generalized gradient approximation exchange-correlation functional proposed by Perdew, Burke, and Ernzerhof was combined with the double numerical basis set plus polarization functions. A supercell slab consisting of a $4 \times$ 4 surface unit cell with three atomic (111) surface layers was adopted, with the bottom two layers fixed at the corresponding bulk positions and the top layers allowed to relax during geometry optimizations. The slab was separated by a vacuum space with a height of $30 \AA . E_{\mathrm{ad}}$ was defined by the equation $E_{\mathrm{ad}}=E_{\mathrm{FA}}+$ $E_{\text {slab }}-E_{\mathrm{FA} / \text { slab}}$, where $E_{\mathrm{FA}}$ is the total energy of free $\mathrm{FA}, E_{\text {slab }}$ is the total energy of the bare slab model, and $E_{\mathrm{FA} / \mathrm{slab}}$ is the total energy of the FA-slab system.

DFT calculations to obtain total energy, harmonic frequency, and Gibbs free energy values for the reactants, TSs, and products for the five reactions were performed using the Gaussian 09 program. In each case, TSs were first characterized, after which an intrinsic reaction coordinate analysis was performed to seamlessly connect the TSs to the reactants and products ${ }^{68}$. The Becke threeparameter Lee-Yang-Parr hybrid-type functional ${ }^{69}$ was used, which was implemented in the Gaussian 09 program, and the Los Alamos ECP 70 with the double- $\zeta$ valence basis ${ }^{71}$ was employed for the basis sets of $\mathrm{Pd}$ and $\mathrm{Ag}$ atoms, while the 6-311 G(d,p) basis sets were used for $\mathrm{H}, \mathrm{C}, \mathrm{N}$, and $\mathrm{O}$ atoms. The $\mathrm{T}$ values and reaction rates were calculated using both $\mathrm{Pd}_{2} \mathrm{Ag}_{2}$ and $\mathrm{Pd}_{7} \mathrm{Ag}_{6}$ cluster models.

\section{Data availability}

All data generated and analyzed during this study are included in this article and its Supplementary Information or are available from the corresponding authors upon reasonable request.

Received: 4 June 2019 Accepted: 12 August 2019

Published online: 25 September 2019

\section{References}

1. Vértes, A., Nagy, S., Klencsár, Z. \& Lovas, R. G. Handbook of Nuclear Chemistry (Kluwer Academic Publishers, 2003)

2. Sattler, A. Hydrogen/deuterium (H/D) exchange catalysis in alkanes. ACS Catal. 8, 2296-2312 (2018)

3. Jones, W. D. Isotope effects in $\mathrm{C}-\mathrm{H}$ bond activation reactions by transition metals. Acc. Chem. Res. 36, 140-146 (2003).

4. Gómez-Gallego, M. \& Sierra, M. A. Kinetic isotope effects in the study of organometallic reaction mechanisms. Chem. Rev. 111, 4857-4963 (2011).

5. Atzrodt, J., Derdau, V., Kerr, W. J. \& Reid, M. Deuterium- and tritium-labelled compounds: applications in the life sciences. Angew. Chem. Int. Ed. 57, 1758-1784 (2018).

6. Junk, T. \& Catallo, W. J. Hydrogen isotope exchange reactions involving $\mathrm{C}-\mathrm{H}$ (D, T) bonds. Chem. Soc. Rev. 26, 401-406 (1997).

7. Mann, W. B., Newell, W. C. \& Rankine Alexander, O. The exchanges of energy between a platinum surface and hydrogen and deuterium molecules. Proc. $R$. Soc. Lond. Ser. A 158, 397-403 (1937).

8. Azua, A., Sanz, S. \& Peris, E. Water-soluble Ir III N-heterocyclic carbene based catalysts for the reduction of $\mathrm{CO}_{2}$ to formate by transfer hydrogenation and the deuteration of aryl amines in water. Chem. Eur. J. 17, 3963-3967 (2011).

9. Carriker, J. L., Wagenknecht, P. S., Hosseini, M. A. \& Fleming, P. E. Transition metal catalyzed $\mathrm{D}_{2} / \mathrm{H}_{2} \mathrm{O}$ exchange: distinguishing between the single and double exchange pathways. J. Mol. Catal. A 267, 218-223 (2007).

10. Kovács, G., Nádasdi, L., Laurenczy, G. \& Joó, F. Aqueous organometallic catalysis. Isotope exchange reactions in $\mathrm{H}_{2}-\mathrm{D}_{2} \mathrm{O}$ and $\mathrm{D}_{2}-\mathrm{H}_{2} \mathrm{O}$ systems catalyzed by water-soluble Rh- and Ru-phosphine complexes. Green. Chem. 5, 213-217 (2003).

11. Jalón, F. A. et al. Facile $\mathrm{Ru}-\mathrm{H}_{2}$ heterolytic activation and intramolecular proton transfer assisted by basic $\mathrm{n}$-centers in the ligands. J. Am. Chem. Soc. 127, 15364-15365 (2005)

12. Grundler, P. V. et al. Kinetic studies on the first dihydrogen aquacomplex, $[R u$ $\left.\left(\mathrm{H}_{2}\right)\left(\mathrm{H}^{2} \mathrm{O}\right)_{5}\right]^{2+}$ : formation under $\mathrm{H}_{2}$ pressure and catalytic $\mathrm{H} / \mathrm{D}$ isotope exchange in water. Inorg. Chim. Acta 359, 1795-1806 (2006).

13. Vignais, P. M. H/D exchange reactions and mechanistic aspects of the hydrogenases. Coord. Chem. Rev. 249, 1677-1690 (2005).

14. Kurita, T. et al. Facile and convenient method of deuterium gas generation using a $\mathrm{Pd} / \mathrm{C}$-catalyzed $\mathrm{H}_{2}-\mathrm{D}_{2}$ exchange reaction and its application to synthesis of deuterium-labeled compounds. Chem. Eur. J. 14, 3371-3379 (2008).

15. Enomoto, A., Kajita, S. \& Fujita, K.-i Convenient method for the production of deuterium gas catalyzed by an iridium complex and its application to the deuteration of organic compounds. Chem. Lett. 48, 106-109 (2019).

16. Eppinger, J. \& Huang, K.-W. Formic acid as a hydrogen energy carrier. ACS Energy Lett. 2, 188-195 (2017).

17. Grasemann, M. \& Laurenczy, G. Formic acid as a hydrogen source-recent developments and future trends. Energy Environ. Sci. 5, 8171-8181 (2012).

18. Joó, F. Breakthroughs in hydrogen storage-formic acid as a sustainable storage material for hydrogen. ChemSusChem 1, 805-808 (2008).

19. Bulushev, D. A. \& Ross, J. R. H. Heterogeneous catalysts for hydrogenation of $\mathrm{CO}_{2}$ and bicarbonates to formic acid and formates. Catal. Rev. 60, 566-593 (2018).

20. Gunasekar, G. H., Park, K., Jung, K.-D. \& Yoon, S. Recent developments in the catalytic hydrogenation of $\mathrm{CO}_{2}$ to formic acid/formate using heterogeneous catalysts. Inorg. Chem. Front 3, 882-895 (2016).

21. Mori, K., Sano, T., Kobayashi, H. \& Yamashita, H. Surface engineering of a supported PdAg catalyst for hydrogenation of $\mathrm{CO}_{2}$ to formic acid: elucidating the active Pd atoms in alloy nanoparticles. J. Am. Chem. Soc. 140, 8902-8909 (2018).

22. Mori, K., Taga, T. \& Yamashita, H. Isolated single-atomic Ru catalyst bound on a layered double hydroxide for hydrogenation of $\mathrm{CO}_{2}$ to formic acid. ACS Catal. 7, 3147-3151 (2017).

23. Singh, A. K., Singh, S. \& Kumar, A. Hydrogen energy future with formic acid: a renewable chemical hydrogen storage system. Catal. Sci. Technol. 6, 12-40 (2016).

24. Mellmann, D., Sponholz, P., Junge, H. \& Beller, M. Formic acid as a hydrogen storage material - development of homogeneous catalysts for selective hydrogen release. Chem. Soc. Rev. 45, 3954-3988 (2016).

25. Gao, Y., Kuncheria, J. K., Jenkins, H. A., Puddephatt, R. J. \& Yap, G. P. A. The interconversion of formic acid and hydrogen/carbon dioxide using a binuclear ruthenium complex catalyst. J. Chem. Soc. Dalton Trans. https://doi.org/ 10.1039/B004234J, 3212-3217 (2000).

26. Fukuzumi, S., Kobayashi, T. \& Suenobu, T. Efficient catalytic decomposition of formic acid for the selective generation of $\mathrm{H}_{2}$ and $\mathrm{H} / \mathrm{D}$ exchange with a water-soluble rhodium complex in aqueous solution. ChemSusChem 1, 827-834 (2008)

27. Fukuzumi, S., Kobayashi, T. \& Suenobu, T. Unusually large tunneling effect on highly efficient generation of hydrogen and hydrogen isotopes in $\mathrm{pH}$-selective decomposition of formic acid catalyzed by a heterodinuclear iridium-ruthenium complex in water. J. Am. Chem. Soc. 132, 1496-1497 (2010).

28. Wang, W.-H. et al. Highly efficient $\mathrm{D}_{2}$ generation by dehydrogenation of formic acid in $\mathrm{D}_{2} \mathrm{O}$ through $\mathrm{H}^{+} / \mathrm{D}^{+}$exchange on an iridium catalyst: application to the synthesis of deuterated compounds by transfer deuterogenation. Chem. Eur. J. 18, 9397-9404 (2012).

29. Mori, K., Dojo, M. \& Yamashita, H. Pd and Pd-Ag nanoparticles within a macroreticular basic resin: an efficient catalyst for hydrogen production from formic acid decomposition. ACS Catal. 3, 1114-1119 (2013).

30. Mori, K., Tanaka, H., Dojo, M., Yoshizawa, K. \& Yamashita, H. Synergic catalysis of $\mathrm{PdCu}$ alloy nanoparticles within a macroreticular basic resin for hydrogen production from formic acid. Chem. Eur. J. 21, 12085-12092 (2015).

31. Mori, K., Naka, K., Masuda, S., Miyawaki, K. \& Yamashita, H. Palladium copper chromium ternary nanoparticles constructed in situ within a basic resin: enhanced activity in the dehydrogenation of formic acid. ChemCatChem 9, 3456-3462 (2017).

32. Mori, K. et al. Phenylamine-functionalized mesoporous silica supported PdAg nanoparticles: a dual heterogeneous catalyst for formic acid/ $\mathrm{CO}_{2}$-mediated chemical hydrogen delivery/storage. Chem. Commun. 53, 4677-4680 (2017).

33. Masuda, S., Mori, K., Futamura, Y. \& Yamashita, H. PdAg nanoparticles supported on functionalized mesoporous carbon: promotional effect of surface 
amine groups in reversible hydrogen delivery/storage mediated by formic acid/CO 2 . ACS Catal. 8, 2277-2285 (2018).

34. Martis, M., Mori, K., Fujiwara, K., Ahn, W.-S. \& Yamashita, H. Aminefunctionalized MIL-125 with imbedded palladium nanoparticles as an efficient catalyst for dehydrogenation of formic acid at ambient temperature. J. Phys. Chem. C 117, 22805-22810 (2013).

35. Navlani-García, M., Mori, K., Nozaki, A., Kuwahara, Y. \& Yamashita, H. Investigation of size sensitivity in the hydrogen production from formic acid over carbon-supported Pd nanoparticles. ChemistrySelect 1, 1879-1886 (2016).

36. Tedsree, K. et al. Hydrogen production from formic acid decomposition at room temperature using a Ag-Pd core-shell nanocatalyst. Nat. Nanotechnol. 6, 302-307 (2011).

37. Qin, Y.-1, Wang, J., Meng, F.-z, Wang, L.-m \& Zhang, X.-b Efficient PdNi and PdNi@Pd-catalyzed hydrogen generation via formic acid decomposition at room temperature. Chem. Commun. 49, 10028-10030 (2013).

38. Ojeda, M. \& Iglesia, E. Formic acid dehydrogenation on Au-based catalysts at near-ambient temperatures. Angew. Chem. Int. Ed. 48, 4800-4803 (2009).

39. Bercaw, J. E., Chen, G. S., Labinger, J. A. \& Lin, B.-L. Hydrogen tunneling in protonolysis of platinum(ii) and palladium(ii) methyl complexes: mechanistic implications. J. Am. Chem. Soc. 130, 17654-17655 (2008).

40. McMahon, R. J. Chemical reactions involving quantum tunneling. Science 299, 833-834 (2003).

41. Meisner, J. \& Kästner, J. Atom tunneling in chemistry. Angew. Chem. Int. Ed. 55, 5400-5413 (2016).

42. Schreiner, P. R. Tunneling control of chemical reactions: the third reactivity paradigm. J. Am. Chem. Soc. 139, 15276-15283 (2017).

43. Pan, Z., Horner, J. H. \& Newcomb, M. Tunneling in C-H oxidation reactions by an oxoiron(iv) porphyrin radical cation: direct measurements of very large H/D kinetic isotope effects. J. Am. Chem. Soc. 130, 7776-7777 (2008).

44. Slaughter, L. M., Wolczanski, P. T., Klinckman, T. R. \& Cundari, T. R. Interand intramolecular experimental and calculated equilibrium isotope effects for $(\text { silox })_{2}\left(t \mathrm{Bu}_{3} \mathrm{SiND}\right) \mathrm{TiR}+\mathrm{RH}\left(\right.$ silox $\left.=t \mathrm{Bu}_{3} \mathrm{SiO}\right)$ : inferred kinetic isotope effects for $\mathrm{RH} / \mathrm{D}$ addition to transient (silox) ${ }_{2} \mathrm{TiNSitBu}_{3}$. J. Am. Chem. Soc. 122, 7953-7975 (2000).

45. Bercaw, J. E., Chen, G. S., Labinger, J. A. \& Lin, B.-L. Protonolysis of platinum (II) and palladium(II) methyl complexes: a combined experimental and theoretical investigation. Organometallics 29, 4354-4359 (2010).

46. Lauderdale, J. G. \& Truhlar, D. G. Large tunneling effects in the migration of chemisorbed hydrogen on a metal. J. Am. Chem. Soc. 107, 4590-4591 (1985).

47. German, E. D., Abir, H. \& Sheintuch, M. A tunnel model for activated hydrogen dissociation on metal surfaces. J. Phys. Chem. C 117, 7475-7486 (2013).

48. Hakanoglu, C., Hawkins, J. M., Asthagiri, A. \& Weaver, J. F. Strong kinetic isotope effect in the dissociative chemisorption of $\mathrm{H}_{2}$ on a $\mathrm{PdO}(101)$ thin film. J. Phys. Chem. C 114, 11485-11497 (2010).

49. Lauhon, L. J. \& Ho, W. Direct observation of the quantum tunneling of single hydrogen atoms with a scanning tunneling microscope. Phys. Rev. Lett. 85, 4566-4569 (2000).

50. McIntosh, E. M., Wikfeldt, K. T., Ellis, J., Michaelides, A. \& Allison, W. Quantum effects in the diffusion of hydrogen on $\mathrm{Ru}(0001)$. J. Phys. Chem. Lett. 4, 1565-1569 (2013).

51. Suleimanov, Y. V. Surface diffusion of hydrogen on $\mathrm{Ni}(100)$ from ring polymer molecular dynamics. J. Phys. Chem. C 116, 11141-11153 (2012)

52. Sierka, M. \& Sauer, J. Proton mobility in chabazite, faujasite, and ZSM-5 zeolite catalysts. Comparison based on ab initio calculations. J. Phys. Chem. B 105, 1603-1613 (2001)

53. Janik, M. J., Davis, R. J. \& Neurock, M. Anhydrous and water-assisted proton mobility in phosphotungstic acid. J. Am. Chem. Soc. 127, 5238-5245 (2005).

54. Volpi, A. \& Clary, D. C. Theoretical investigation of the surface reaction N $(\mathrm{ads})+\mathrm{H}(\mathrm{ads}) \rightarrow \mathrm{NH}(\mathrm{ads})$ on $\mathrm{Ru}(0001)$ using density functional calculations, variational transition-state theory, and semiclassical tunneling method. $J$. Phys. Chem. B 108, 336-345 (2004).

55. German, E. D. \& Sheintuch, M. Theoretical study of catalytic CO oxidation on (111) metal surfaces: calculating rate constants that account for tunnel effect. J. Phys. Chem. C 111, 9184-9193 (2007).

56. Mudiyanselage, K., Trenary, M. \& Meyer, R. J. Kinetics of NH formation and dissociation on Pt(111). J. Phys. Chem. C 111, 7127-7136 (2007).

57. Tautermann, C. S., Sturdy, Y. K. \& Clary, D. C. Reaction rates of all hydrogenation steps in ammonia synthesis over a $\mathrm{Ru}(0001)$ surface. J. Catal. 244, 199-207 (2006).

58. Waluyo, I., Ren, Y. \& Trenary, M. Observation of tunneling in the hydrogenation of atomic nitrogen on the $\mathrm{Ru}(001)$ surface to form NH. J. Phys. Chem. Lett. 4, 3779-3786 (2013).

59. Skodje, R. T., Truhlar, D. G. \& Garrett, B. C. A general small-curvature approximation for transition-state-theory transmission coefficients. J. Phys. Chem. 85, 3019-3023 (1981).

60. Truhlar, D. G. \& Garrett, B. C. Reduced mass in the one-dimensional treatment of tunneling. J. Phys. Chem. A 107, 4006-4007 (2003).
61. Garrett, B. C., Abusalbi, N., Kouri, D. J. \& Truhlar, D. G. Test of variational transition state theory and the least-action approximation for multidimensional tunneling probabilities against accurate quantal rate constants for a collinear reaction involving tunneling into an excited state. J. Chem. Phys. 83, 2252-2258 (1985).

62. Liu, Y. P., Lu, D. H., Gonzalez-Lafont, A., Truhlar, D. G. \& Garrett, B. C. Direct dynamics calculation of the kinetic isotope effect for an organic hydrogen-transfer reaction, including corner-cutting tunneling in 21 dimensions. J. Am. Chem. Soc. 115, 7806-7817 (1993).

63. Fermann, J. T. \& Auerbach, S. Modeling proton mobility in acidic zeolite clusters: II. Room temperature tunneling effects from semiclassical rate theory. J. Chem. Phys. 112, 6787-6794 (2000)

64. Jewell, A. D. et al. Quantum tunneling enabled self-assembly of hydrogen atoms on $\mathrm{Cu}(111)$. ACS Nano 6, 10115-10121 (2012).

65. Bhatia, B. \& Sholl, D. S. Quantitative assessment of hydrogen diffusion by activated hopping and quantum tunneling in ordered intermetallics. Phys. Rev. B 72, 224302 (2005).

66. Ogo, S. et al. A Functional [NiFe]hydrogenase mimic that catalyzes electron and hydride transfer from $\mathrm{H}_{2}$. Science 339, 682-684 (2013).

67. Delley, B. From molecules to solids with the $\mathrm{DMol}^{3}$ approach. J. Chem. Phys. 113, 7756-7764 (2000).

68. Fukui, K., Kato, S. \& Fujimoto, H. Constituent analysis of the potential gradient along a reaction coordinate. Method and an application to methane + tritium reaction. J. Am. Chem. Soc. 97, 1-7 (1975).

69. Becke, A. D. Density-functional thermochemistry. III. The role of exact exchange. J. Chem. Phys. 98, 56485652 (1993).

70. Hay, P. J. \& Wadt, W. R. Ab initio effective core potentials for molecular calculations. Potentials for the transition metal atoms Sc to Hg. J. Chem. Phys. 82, 270-283 (1985).

71. Dunning, T. H. \& Hay, P. J. in Modern Theoretical Chemistry, Vol. 3 (ed. Henry, F. S.) 1-28 (Plenum Press, 1976).

\section{Acknowledgements}

The present work was supported by JST-PRESTO (JPMJPR1544). The part of this work was supported by Grants-in-Aid for Scientific Research (Nos. 26220911 and A18H020740) from the Japan Society for the Promotion of Science (JSPS) and MEXT. This work was also partially supported by MEXT program Elements Strategy Initiative to Form Core Research Center.

\section{Author contributions}

K.M. and H.Y. initiated and supervised the project and conceived the experiment. Y.F performed the catalyst preparation and characterization. S.M. helped the sample preparation. H.K. performed DFT calculations. K.M. wrote the manuscript. The manuscript was written through the discussion with all authors. All authors have given approval to the final version of the manuscript.

\section{Additional information}

Supplementary Information accompanies this paper at https://doi.org/10.1038/s41467019-12018-7.

Competing interests: The authors declare no competing interests.

Reprints and permission information is available online at http://npg.nature.com/ reprintsandpermissions/

Peer review information: Nature Communications thanks the anonymous reviewers for their contribution to the peer review of this work. Peer reviewer reports are available.

Publisher's note: Springer Nature remains neutral with regard to jurisdictional claims in published maps and institutional affiliations.

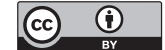

Open Access This article is licensed under a Creative Commons Attribution 4.0 International License, which permits use, sharing, adaptation, distribution and reproduction in any medium or format, as long as you give appropriate credit to the original author(s) and the source, provide a link to the Creative Commons license, and indicate if changes were made. The images or other third party material in this article are included in the article's Creative Commons license, unless indicated otherwise in a credit line to the material. If material is not included in the article's Creative Commons license and your intended use is not permitted by statutory regulation or exceeds the permitted use, you will need to obtain permission directly from the copyright holder. To view a copy of this license, visit http://creativecommons.org/licenses/by/4.0/. 